\title{
The 2014 AHA/ACC valve disease guideline: new stages of disease, new treatment options, and a call for earlier intervention
}

\author{
Julia Mascherbauer
}

Published online: 15 August 2014

(C) Springer-Verlag Wien 2014

The American Heart Association (AHA) and the American College of Cardiology (ACC) have issued a new joint guideline for the treatment of adults with acquired valvular heart disease, which includes substantial changes of the previous 2008 guideline.

Several important changes and additions are listed:

1. Stages of heart valve disease. For each of the cardiac valves, the guideline describes stages of disease, similar to the classification of heart failure stages based on valve anatomy, valve hemodynamics, hemodynamic consequences, and symptoms: $\mathrm{A}$, at risk; $\mathrm{B}$, progressive; $\mathrm{C}$, asymptomatic severe; and D, symptomatic severe.

2. Cardiac magnetic resonance imaging. The new guideline gives cardiac magnetic resonance imaging a Class I recommendation for the assessment of patients with chronic severe aortic and mitral regurgitation (MR).

3. Exercise testing. Increased emphasis (Class IIa) is placed on the use of exercise testing in the evaluation of asymptomatic patients with severe heart valve disease (particularly aortic stenosis (AS) and primary MR).

4. Aortic stenosis. Changes were made in the characterization of AS and indications for valve replacement:

4.1 Characterization of AS:

a. "Very severe" AS is defined as aortic $\mathrm{V}_{\max } \geq 5 \mathrm{~m} / \mathrm{s}$ or mean gradient $\geq 60 \mathrm{mmHg}$.

b. Symptomatic severe AS is subdivided into

Ao. Univ.-Prof. Dr. J. Mascherbauer $(\bowtie)$

Universitätsklinik für Innere Medizin II,

Medizinische Universität Wien,

Währinger Gürtel 18-20,

1090 Vienna, Austria

e-mail: julia.mascherbauer@meduniwien.ac.at
- High gradient AS $\left(\mathrm{V}_{\max } \geq 4 \mathrm{~m} / \mathrm{s}\right.$ or mean gradient $\geq 40 \mathrm{mmHg}$ ),

- Low-flow low-gradient AS with reduced left ventricular ejection fraction

- Low-flow low-gradient AS with normal left ventricular ejection fraction (paradoxical low-flow low-gradient AS)

4.2 Indications for valve replacement are expanded to include:

a. Asymptomatic "very severe" AS and low surgical risk (Class IIa)

b. Asymptomatic severe AS and decreased exercise tolerance or exercise-related decrease in blood pressure (Class IIa)

c. Symptomatic patients with low-flow low-gradient severe AS and normal left ventricular ejection fraction if clinical, hemodynamic, and anatomic data support valve obstruction as the likely cause of symptoms (Class IIa).

5. Heart Valve Teams, Heart Valve Centers of Excellence. Like the 2012 European valve guidelines, the new AHA/ACC document also emphasizes the role of multidisciplinary teams and defines the important role of both Heart Valve Teams and Heart Valve Centers of Excellence.

6. Evaluation of surgical and interventional risk. A risk score is proposed that uses the following:

- STS (society of thoracic surgeons risk score) predicted risk of mortality

- Frailty, measured with accepted indices

- Major organ system compromise not to be improved postoperatively

- Procedure-specific impediment 
The guideline urges clinicians not to rely on a single parameter, but to consider all relevant factors constituting risk for each specific patient, and to involve patients in decision-making.

7. Transcatheter aortic valve implantation (TAVI). Surgical aortic valve replacement (AVR) remains the intervention of choice for patients with low or intermediate operative risk (Class I) because longterm data are still unavailable regarding longevity of valves placed by the transcatheter approach.

Both AHA/ACC and European guidelines give TAVI a Class I recommendation for high-risk patients with AS who are not suitable candidates for surgical AVR. TAVI is recommended as a "reasonable alternative" to surgical AVR in patients who are at high surgical risk but who are otherwise suitable for surgery (Class IIa in both sets of guidelines).

8. Transcatheter mitral valve repair. The new guideline, as well as the 2012 European document, note a US Food and Drug Administration-approved device for transcatheter mitral valve repair. Use of this device may be an option for severely symptomatic patients with chronic severe primary MR (stage D), favorable anatomy, reasonable life expectancy, and prohibitive surgical risk (Class IIb).

9. "Prophylactic" mitral valve repair. According to the new guideline "prophylactic" mitral valve repair is reasonable in patients with asymptomatic severe chronic primary MR and preserved left ventricular (LV) function when performed at a Heart Valve Center of Excellence and when the likelihood of successful and durable repair without residual MR is $>95 \%$ and operative mortality risk is $<1 \%$ (Class IIa).

10. Management of secondary MR. The treatment of chronic secondary MR involves treatment of the underlying cardiomyopathy (Class I) and cardiac resynchronization therapy if indicated (Class I). Intervention for chronic secondary MR is reason- able at the time of coronary artery bypass grafting or other cardiac surgery if MR is severe (Class IIa) and not unreasonable if MR is moderate (Class IIb).

11. Low-molecular-weight heparin (LMWH) "bridging" of patients with mechanical heart valve prostheses. LMWH "bridging" is now considered appropriate (Class I) among patients with a mechanical valve at high risk of thrombosis when warfarin needs to be interrupted (previously Class IIb).

\section{Clinical implications and perspective}

The new AHA/ACC guideline is a very detailed and comprehensive document, covering many new aspects of acquired adult valvular heart disease. It offers recommendations for the management of native valve disease, prosthetic valves, infective endocarditis, and pregnancy of patients with valvular heart disease. Questions related to cardiac and noncardiac surgery are also covered. The guideline recommends a new staging algorithm and a risk-scoring system. Several additions have been made in answer to new treatment options such as minimally invasive surgical and transcatheter valve interventions. The new guideline also reflects a shift toward intervening earlier in the course of the disease and provides a new system for classifying patient risk prior to a procedure. Like the European guideline published in 2012, the AHA/ ACC document emphasizes the role of multidisciplinary teams and defines the important role of Heart Valve Teams and Heart Valve Centers of Excellence.

\section{Reference}

1. Nishimura RA, Otto CM, Bonow RO, Carabello BA, Erwin JP 3rd, Guyton RA, O'Gara PT, Ruiz CE, Skubas NJ, Sorajja P, Sundt TM 3rd, Thomas JD; ACC/AHA Task Force Members. Circulation. 2014 Jun 10;129(23):e521-643. doi: 10.1161/ CIR.0000000000000031. Epub 2014 Mar 3. No abstract available. 